\title{
HISTERECTOMÍA VAGINAL SIN PROLAPSO: ESTUDIO DE COHORTE. MEDELLÍN (COLOMBIA) 2008-2010
}

\section{Vaginal hysterectomy without prolapse: a 2008-2010 colhort study in Medellín (Colombia)}

José Luis Correa-Ochoa, M.D. *, Jorge Alberto Tirado-Mejía, M.D. *, Juan Fernando Mejía-Zúñiga, M.D.**, Marcela Tirado-Hernández, M.D.**, Edwin Alcides Gómez-Ibarra, M.D.**, Adriana Arango-Martínez, M.D., MSc***

Recibido: junio 4/10 - Aceptado: febrero 21/11

\section{RESUMEN}

Objetivo: describir los resultados posquirúrgicos de la histerectomía vaginal sin prolapso uterino.

Materiales y métodos: estudio de cohorte de pacientes, a quienes se les practicó histerectomía vaginal sin prolapso (HSVP) por patología benigna uterina, utilizando la técnica de Heaney modificada y técnicas de morcelamiento uterino en la Clínica Medellín - Antioquia, entre septiembre de 2008 y febrero de 2010. Se realizó muestreo secuencial estricto. Se estimó un tamaño de muestra de 84 pacientes. Se midieron las características sociodemográficas, características del útero, diagnóstico preoperatorio, tiempo quirúrgico, comorbilidades, y complicaciones.

Resultados: fueron intervenidos 84 pacientes, de los cuales un $8,33 \%$ presentaron complicaciones (n: 7/84), el 3,6\% de estas, fueron intraoperatorias (2 lesiones vesicales y 1 lesión rectal) y el 4,76\% posoperatorias (1 absceso pélvico con dehiscencia secundaria de la herida, 1 sepsis, 2 granulomas del muñón vaginal). También, fueron encontrados 9 casos de infección del tracto urinario (ITU) (10,71\%).

\footnotetext{
* Ginecólogo Clínica Medellín. Medellín (Colombia). Correo electrónico: perseojlcorreao@gmail.com

** Residente de Ginecología y Obstetricia Universidad CES. Medellín (Colombia).

*** Ginecóloga - MSc Epidemiología - Profesor Universidad CES. Medellín (Colombia).
}

Conclusión: La HVSP es una técnica quirúrgica que en manos expertas y con instrumental quirúrgico adecuado, surge como alternativa para el manejo de la patología uterina benigna.

Palabras clave: histerectomía vaginal, prolapso uterino, complicaciones intraoperatorias, complicaciones posoperatorias.

\section{SUMMARY}

Objective: describing the post-surgical results of vaginal hysterectomy $(\mathrm{VH})$ without uterine prolapse. Materials and methods: this was a cohort study of patients who had undergone vaginal hysterectomy without prolapse (VHWP) for benign uterine pathology using a modified Heaney technique and uterine morcellation techniques in the Clínica Medellín between September 2008 and February 2010. Strict sequential sampling was done. Patient sample size was estimated at 84. Socio-demographic characteristics, uttering characteristics, pre-operation diagnosis, time in surgery, comorbidities and complications were measured.

Results: 84 patients were operated on; complications occurred in $8.33 \%$ of them (n: 7/84), 3.6\% were intra-operation (2 vesical lesions and 1 rectal lesion) and $4.76 \%$ were post-operation ( 1 pelvic abscess with secondary dehiscence of the wound, 1 sepsis, 2 granulomas of the vaginal cuff). There were 9 cases of urinary tract infection (UTI) (10.71\%). 
Conclusion: VHWP emerges as an alternative for surgical treatment of benign uterine diseases when performed by expert hands and with the appropriate surgical instrumentals.

Key words: vaginal hysterectomy, uterine prolapse, intra-operation complications, post-operation complications.

\section{INTRODUCCIÓN}

Se define a la histerectomía vaginal sin prolapso (HVSP), a la técnica quirúrgica que permite la extracción del útero a través de la vagina, sin necesidad de realizar incisiones en la pared abdominal y en ausencia de prolapso uterino.

Inicialmente la histerectomía se realizó por vía vaginal, exclusivamente en casos de prolapso, técnica que precedió por siglos a la histerectomía abdominal. Fue descrita por primera vez en el antiguo Egipto por Soranus de Ephesus. ${ }^{1}$ Históricamente, el acceso a la pelvis por vía vaginal ha sido patrimonio exclusivo de los ginecólogos y se espera que el cirujano ginecológico la domine a la perfección. ${ }^{2}$ A mediados del siglo XX, se desestímulo el uso de esta vía en favor de la abdominal pues permitía una inspección ocular directa del campo operatorio y una mejor protección de los uréteres entre otros argumentos. ${ }^{2}$ Sin embargo, algunas escuelas de cirujanos de piso pélvico en varias regiones del mundo, se dedicaron a rescatar, perfeccionar y divulgar la técnica vaginal con un resurgimiento de esta en las dos últimas décadas con buenos resultados. ${ }^{3}$ Hoy en día se utiliza la técnica de HVSP de Heaney ${ }^{4}$ con las modificaciones introducidas por la escuela brasileña de Londrina. ${ }^{5}$ En general, la vía vaginal está indicada en patología benigna de útero, aunque es descrita como una técnica radical para el cáncer de cérvix uterino (F. Schauta 1910) ${ }^{6}$

Si bien, el uso de la HVSP aún no se generaliza, hay estudios que informan que esta técnica tiene ventajas sobre la técnica laparoscópica y la vía abdominal, en cuanto a menor tiempo operatorio, menores tasas de complicaciones, menores costos dados por un menor tiempo de incapacidad y de hospitalización. También se ha descrito una curva de aprendizaje más corta que la laparoscópica y sin los riegos inherentes a la introducción de los trocares y el neumoperitoneo. ${ }^{7-11}$

No se ha realizado una evaluación en el medio local de esta técnica. Por lo tanto, el objetivo del presente trabajo es describir la frecuencia y tipo de complicaciones asociadas a la HVSP.

\section{MATERIALES Y MÉTODOS}

Estudio de una cohorte de pacientes a quienes se les realizó HVSP en el Servicio de Ginecología de la Clínica Medellín, institución privada, que presta servicios de alta complejidad a pacientes del régimen subsidiado y contributivo del sistema de seguridad social y pacientes privados provenientes de la ciudad del Departamento de Antioquia, Colombia. Se incluyeron pacientes con patología uterina benigna sin prolapso uterino, quienes firmaron el consentimiento informado. Se excluyeron pacientes en embarazo, con amenorrea sin diagnóstico, sospecha de síndrome adherencial pélvico severo de cualquier origen, antecedente de peritonitis de cualquier origen, infección activa del tracto genital, antecedente de absceso tuboovárico, antecedente o sospecha de endometriosis severa, presencia de masa anexial, radioterapia local, vagina infundibuliforme, lesiones vaginales obstructivas, lesiones de cadera, úteros de más de $1.300 \mathrm{~g}$ (tamaño similar a 16 semanas de gestación), tumores anexiales o enfermedad maligna avanzada.

Se realizó un muestreo secuencial estricto y el cálculo de un tamaño muestral de 84 pacientes, con base en una frecuencia esperada de complicaciones del 6\%, ${ }^{12}$ un margen de error del 5\%, y una confianza del 95\%.

\section{Procedimiento}

Preparación de la paciente: cerclaje anal previo al inicio de la cirugía, con hilo de seda 2/0 para proporcionar un campo operatorio lo más limpio posible. Evacuación parcial de la vejiga antes de 
iniciar el procedimiento, ya que en caso de lesión de ésta durante el procedimiento, la salida de orina la hará más evidente. Se utiliza profilaxis antibiótica. Se coloca a la paciente en posición de litotomía forzada, utilizando unos estribos que permitan elevar los pies de la paciente y avanzando la región glútea un poco por fuera del borde de la camilla, de modo que todo el grupo quirúrgico se ubique al frente del periné, en un mismo plano.

Técnica de Heaney modificada: $:^{5}$ el instrumental es fundamental y se caracteriza por ser específico, adecuado, largo, fuerte y atraumático. Sus principales objetivos son: la tracción de los tejidos, la optimización del campo operatorio, proporcionando un avance en profundidad más fácil y protegiendo la vejiga, los uréteres y el recto, asegurando una técnica quirúrgica efectiva y segura. Este instrumental está constituido por valvas de separación (Breisky y Steiner-Auvard o valvas pesadas con lámina corta y lámina larga), pinzas para prensión (Z Clamp curvo y semicurvo) y pinzas para tracción de tejidos con dientes agudos (Lahey).

Se describen 6 pasos progresivos: 1) incisión anterior del cuello, separación de la vejiga y apertura de la hoja anterior del peritoneo, 2) incisión posterior del cuello y apertura del fondo de saco de Douglas, se ingresa a la cavidad pélvica por los fondos de saco anterior y posterior para ubicar las valvas de separación (Breisky anteriormente y la Steiner-Auvard con lámina larga posteriormente), con lo cual se protege la vejiga, uréteres y el recto. Se conserva una porción de mucosa vaginal bilateralmente sin incidir, para mantener la inserción de los ligamentos de sostén de la vaginal (ligamentos uterosacros y cardinales) unidos a ella, 3) sección y ligadura del complejo de ligamento uterosacro/ cardinal y anclaje de la cúpula vaginal, 4) ligadura de los vasos uterinos, 5) exteriorización del cuerpo uterino y sección de los pedículos superiores. Los úteros con volumen mayor a $280 \mathrm{cc}$ requieren un tratamiento de reducción con alguna de las técnicas de morcelamiento para una extracción exitosa y 6) revisión de hemostasia y cierre de cúpula vaginal.
En todos los casos, el procedimiento fue realizado por el mismo ginecólogo experto y con dos ayudantes quirúrgicos entrenados en la técnica, durante los cuales, se midieron las siguientes variables: indicaciones de cirugía, tiempo de cirugía, estancia hospitalaria, pérdida sanguínea, complicaciones intraoperatorias, posoperatorias inmediatas (ocurridas en las primeras 24 horas), posoperatorias mediatas (ocurridas entre las 24 horas hasta los primeros 21 días), posoperatorias tardías (ocurridas después de 21 días de la cirugía). Estas mediciones fueron realizadas por los investigadores directamente.

\section{Análisis}

Los datos fueron ingresados en el programa Excel y fueron analizados utilizando el programa SPSS $13^{\circledR}$. Se realizó estadística descriptiva. Los datos se presentan como medidas de tendencia central y de dispersión, con los respectivos intervalos para las variables continuas y proporciones para categóricas.

\section{RESULTADOS}

Ingresaron 84 pacientes cuyas características sociodemográficas y clínicas se observan en la tabla $\mathbf{1}$. El tiempo operatorio, estancia y sangrado se observan en la tabla 2. El sangrado intraoperatorio fue de 300 $\mathrm{ml}$ o menos en el 94\% de las pacientes, un 20,2\% de los casos requirió técnicas de morceramiento para la extracción. No se requirieron transfusiones de productos sanguíneos.

En total 7 pacientes presentaron complicaciones $(8,33 \%)$. Tres fueron intraoperatorias $(3,6 \%)$, correspondientes a 2 lesiones de vejiga (2,38\%) y una lesión de recto $(1,19 \%)$. No se presentaron complicaciones inmediatas. Hubo 2 complicaciones mediatas: 1 absceso de cúpula vaginal con dehiscencia secundaria de la herida $(1,19 \%, 1 / 84)$ y 1 caso de sepsis $(1,19 \%, 1 / 84)$, este último en una paciente desnutrida, con hipoproteinemia como factores de riesgo. Finalmente se observaron 2 complicaciones tardías: 2 granulomas de cúpula vaginal (2,38\%). No hubo necesidad en ninguno de los casos 


\begin{tabular}{|c|c|}
\hline Generalidades grupo & $\mathbf{X}$ \\
\hline Edad & $44,3( \pm 5,02) *$ \\
\hline Peso & $64,4( \pm 11,5)^{*}$ \\
\hline IMC & $24,4( \pm 6,61)^{*}$ \\
\hline Diagnóstico preoperatorio & n (\%) \\
\hline Miomatosis uterina & $64(76,1)$ \\
\hline Hemorragia uterina anormal & $28(33,3)$ \\
\hline Miohipertrofia uterina & $8(9,52)$ \\
\hline NIC III & $2(2,38)$ \\
\hline Hiperplasia endometrial & $2(2,38)$ \\
\hline Dolor pélvico crónico & $1(1,19)$ \\
\hline Cistocele & $8(9,52)$ \\
\hline Rectocele & $7(8,33)$ \\
\hline $\begin{array}{l}\text { Incontinencia } \\
\text { urinaria de esfuerzo }\end{array}$ & $6(7,14)$ \\
\hline Comorbilidad & n (\%) \\
\hline Anemia & $25(29,7)$ \\
\hline HTA & $15(17,8)$ \\
\hline Cirugías previas & $12(14,28)$ \\
\hline ICC-hipotiroidismo & $10(11,9)$ \\
\hline Obesidad & $7(8,33)$ \\
\hline Enfermedades pulmonares & $4(4,76)$ \\
\hline Diabetes & $4(4,76)$ \\
\hline Adherencias & $2(2,38)$ \\
\hline Otras & $4(4,76)$ \\
\hline
\end{tabular}

Media ( \pm Desviación estándar)

\section{Tabla 2. Variables del procedimiento}

\begin{tabular}{|l|c|c|}
\hline & Promedio & IC 95\% \\
\hline Tiempo quirúrgico (min.) & 50,3 & $44,48-56,12$ \\
\hline Sangrado operatorio (ml) & 161,2 & $120,61-193,79$ \\
\hline Peso uterino (gramos) & 194,6 & $159,96-229,24$ \\
\hline Estancia hospitalaria (días) & 1,03 & $1-2 *$ \\
\hline
\end{tabular}

Rango

de laparotomía. Hubo 9 casos de infección del tracto urinario (ITU) (10.71\%). En los dos casos de traumatismo intraoperatorio de vejiga no se presentó ITU.

\section{DISCUSIÓN}

Nuestro trabajo muestra una frecuencia del 8\%, relacionada con la técnica quirúrgica y el 10\% relacionada con la instrumentación vesical. La frecuencia de complicaciones en nuestra serie es similar a la informada por otros estudios. ${ }^{12-15}$ Por lo que podemos sugerir que la HVSP es una técnica segura, en concordancia con otros estudios publicados. ${ }^{16-19}$

Por otra parte, al comparar los resultados de de esta investigación en cuanto al operatorio, estos fueron similares a los reportados en otros estudios y con menor tiempo que en la vía laparoscópica. ${ }^{14,19,20-23}$ 
En cuanto a la estancia hospitalaria en el presente estudio concuerda con lo reportado por García en el 2008, ${ }^{24}$ pero difiere de lo reportado por Kovac en 1995 y el 2000, que describe estancias hospitalarias promedio de 2 días. ${ }^{13,15}$

En la segunda mitad del siglo pasado, se consideraba que la histerectomía vaginal sólo se podía realizar si existía prolapso uterino, pero hoy se sabe que el 90-94\% de los casos pueden hacerse en ausencia del prolapso uterino; ${ }^{7}$ inclusive se ha descrito la utilización de esta técnica en úteros grandes, en mujeres con antecedente de cesárea y de cirugía abdomino-pélvica, en pacientes sin partos vaginales e incluso si se requiere oforectomía. ${ }^{3}$

Figuereido considera la vía vaginal como ambulatoria, ya que al $90 \%$ de las pacientes, se les puede dar de alta en las primeras 24 horas. ${ }^{5} \mathrm{La}$ histerectomía vaginal ofrece ventajas adicionales para algunas pacientes con patologías mixtas, ya que facilita la corrección de defectos apicales, del compartimiento anterior y posterior, incontinencia urinaria de esfuerzo y desgarros perineales, todo en un solo acto operatorio por la misma vía. ${ }^{5,9}$

A pesar de los beneficios mencionados de la vía vaginal, ${ }^{2,25}$ la HA sigue siendo la vía más empleada con un porcentaje del 67,9\%, seguida de la vaginal con 21,7\%, y 10,4\% para la laparoscópica según algunos reportes mundiales. ${ }^{1,20,26}$ Los datos obtenidos por algunos estudios, como el meta-análisis de Cochrane en 2006, sugieren que la vaginal es la vía de elección para realizar una histerectomía, siempre que no exista contraindicación absoluta para esta, debido principalmente a una menor morbilidad, menor estancia hospitalaria, y en comparación con la laparoscópica menor costo, y menor tiempo quirúrgico. ${ }^{13,20,26,27}$ Lo anterior, propone a la HV como la ruta más acertada de abordaje quirúrgico, tal como recomiendan el ACOG (Colegio Americano de Obstetras y Ginecólogos) y Kovac.,21,28

Este estudio tiene como limitación no haber tenido un grupo control de comparación, estas observaciones deberán ser confirmadas con la realización de un ensayo clínico controlado que compare la técnica de la HVSP con alguno de los otros tipos de histerectomía.

\section{CONCLUSIÓN}

La HVSP es una técnica quirúrgica que en manos expertas y con instrumental quirúrgico adecuado, surge como alternativa para el manejo de la patología uterina benigna.

\section{AGRADECIMIENTOS}

Especial agradecimiento a la Clínica Medellín y Coomeva EPS por permitirnos llevar a cabo esta descripción de resultados operatorios, al Dr. José Luis Correa por darnos acceso completo a su base de datos y a la Universidad CES por compartir con nosotros el espíritu investigativo.

\section{REFERENCIAS}

1. Kulkarni MM, Rogers RG. Vaginal hysterectomy for benign disease without prolapse. Clin Obstet Gynecol 2010;53:5-16.

2. Sotero-Salgueiro GA, Sosa-Fuertes CG, DomínguezRama A, Martínez-Torena J, Yovarone R, Laborde A. La histerectomía vaginal en útero no prolapsado: una vieja "nueva” opción. Arch Gin Obstet 2002;40:30-7.

3. Salcedo FL. Vaginal hysterectomy in non-prolapsed uteruses: "no scar hysterectomy". Int Urogynecol J Pelvic Floor Diysfunct 2009;20:1009-12.

4. Heaney NS. Vaginal hysterectomy: its indications and technique. Am J Surg 1940;48:284-8.

5. Figuereido O. Histerectomía vaginal em pacientes sem prolapso uterino análise de 150 casos. Rev Bras Ginec Obstet. 2003;19:267-73.

6. Reiffenstuhl G. Platzer W. Operación radical vaginal de Schauta-Americh en el carcinoma de cérvix. En: Reiffenstuhl G. Platzer W. Operaciones por vía vaginal Anatomía Quirúrgica y Técnica Operatoria. Barcelona: Salvat Editores; 1978.

7. Kovac SR. Clinical opinion: guidelines for hysterectomy. Am J Obstet Gynecol 2004;191:635-40.

8. Summitt RL Jr, Stovall TG, Steege J, Lipscomb GH. A multicenter randomized comparison of laparoscopically assisted vaginal hysterectomy and abdominal hysterectomy in abdominal hysterectomy candidates. Obstet Gynecol 1998;92:321-6. 
9. Copenhaver EH. Vaginal hysterectomy: an analysis of indications and complications among 1000 operations. Am J Obstet Gynecol 1962;84:123-128.

10. Lepine LA, Hillis SD, Marchbanks PA, Koonin LM, Morrow B, Kieke BA, et al. Hysterectomy surveillance-United States, 1980-1993. MMWR CDC Surveill Summ 1997;46:1-15.

11. Dorsey JH, Holtz PM, Griffiths RI, McGrath MM, Steinberg EP. Costs and charges associated with three alternative techniques of hysterectomy. N Engl J Med 1996;335:476-82.

12. Agostini A, Bretell F, Cravello 1, Maisonneuver AS, Rager V, Blanc B. Vaginal hysterectomy in nuliparous women without prolapse: a prospective study: BJOG 2003;110:515-8.

13. Kovac SR. Hysterectomy outcomes in patients with similar indications. Obstet Gynecol 2000;95:787-93.

14. Garry R, Fountain J, Mason S, Hawe J, Napp V, Abbot J, et al. The Evaluate study: two parallel randomised trials one comparing laparoscopic with abdominal hysterectomy the other comparing, laparoscopic with vaginal hysterectomy. BMJ 2004;328:129.

15. Kovac SR. Guidelines to determine the route of hysterectomy. Obstet Gynecol 1995;85:18-23.

16. Rodríguez-Hidalgo N. La histerectomía vaginal en pacientes sin prolapso: indicaciones, técnica y recomendaciones. Rev Cubana Obstet Ginecol 1995; 21(1).

17. Occhino J, Gebhart JB. Difficult vaginal hysterectomy. Clin Obstet Gynecol 2010;53:40-50.

18. Benassi L, Rossi T, Kaihura CT, Ricci L, Bedocchi L, Galanti B, et al. Abdominal or vaginal hysterectomy for the enlarged uteri: a randomized Clinical Trial. Am J Obstet Gynecol 2002;187:1561-5.
19. Magos A, Bournas N, Sinha R, Richardson RE, O'Connor H. Vaginal hysterectomy for the large uterus. BJOG 1996;103:246-51.

20. Nieboer T, Johnson N, Lethaby A, Tavender E, Curr E, Garry R. Surgical approach to hysterectomy for benign gynaecological disease. Cochrane Database Syst Rev 2009;(3):CD003677.

21. Kovac S. Transvaginal hysterectomy: rationale and surgical approach. Obstet Gynecol 2004;103:1321-5.

22. Hoffman M, DeCesare S, Kalter C. Abdominal hysterectomy versus transvaginal morcellation for the removal of enlarged uteri. Am J Obstet Gynecol. 1994;171:309-15.

23. De los Ríos J, Castañeda J, Calle G, Serna G, Vásquez R, Arango A, et al. Histerectomía laparoscópica total en la unidad de endoscopia ginecológica de la clínica del Prado, Medellín, (Colombia) 2002 - 2008. Rev Colomb Obstet Ginecol. 2009;60:320-7.

24. García Rodríguez ME, Romero Sánchez RE. Histerectomía vaginal en úteros no prolapsados. A propósito de 300 casos. Rev Cubana Obstet Ginecol 2008;34(1).

25. Paparella P, Sizzi O, Rossetti A, De Benedittis F, Paparella R. Vaginal hysterectomy in generally considered contraindications to vaginal surgery. Arch Gynecol Obstet 2004;270:104-9.

26. Figuereido O, Figueiredo EG, Figueiredo PG, Pelosi MA 3rd, Pelosi MA. Vaginal removal of the benign non prolapsed uterus: experience with 300 consecutive operations. Obstet Gynecol 1999;94:348-51.

27. Johnson N, Barlow D, Lethaby A, Tavender E, Curr L, Garry R. Methods of hysterectomy: systematic review and meta-analysis of randomised controlled trials. BMJ 2005;330:1478.

28. ACOG Committee Opinion No. 444: choosing the route of hysterectomy for benign disease. Obstet Gynecol 2009;114:1156-8. 